\title{
SATISFAÇÃO DOS ACADÊMICOS DE CIÊNCIAS CONTÁBEIS: UM ESTUDO COM EQUAÇÕES ESTRUTURAIS
}

\section{Academics satisfaction of Accounting Sciences: A study with structural equations}

Adhmir Renan Voltolini Gomes

E-mail: adhmir.renan@gmail.com

Mestre em Contabilidade pela Universidade Estadual do Oeste do Paraná; Especialista em Administração Financeira, Contábil e Controladoria pelo Instituto Brasileiro de Pesquisa Socioeconômico (Inbrape); Pesquisador na Universidade Estadual do Oeste do Paraná. https://orcid.org/0000-0003-2089-5924

Rafael Maximiano Ferreira

E-mail: rafamaxf@gmail.com

Mestre em Contabilidade pela Universidade Estadual do Oeste do Paraná; Especialista em Gestão Financeira, Contábil e Controladoria pelo Centro Universitário Univel; Professor no Centro Universitário Univel; Contador na Empresa Germano Zeni Veículos Ltda. (Zeni Motors - Toyota). https://orcid.org/0000-0002-5517-5516

Samuel Lyncon Leandro de Lima E-mail: samuellynconleandro@gmail.com

Mestre em Contabilidade pela Universidade Estadual do Oeste do Paraná; Especialista em Controladoria e Finanças pela Pontifícia Universidade Católica do Paraná; Professor Mediador no Centro Universitário de Maringá. Endereço para contato: Avenida Guedner, 1610, Jardim Aclimação, 87050-900, Maringá, Paraná,

https://orcid.org/0000-0003-3956-3575

Silvana Anita Walter

E-mail: silvanaanita.walter@gmail.com

Doutora em Ciências Contábeis e Administração pela Universidade Regional de Blumenau; Mestra em Administração: Gestão Moderna de Negócios pela Universidade Regional de Blumenau;

Professora no Curso de Administração, no Mestrado Profissional em Administração e no Mestrado Acadêmico em Contabilidade da Universidade Estadual do Oeste do Paraná. https://orcid.org/0000-0003-1684-5465

Artigo recebido em 20 de fevereiro de 2019. Aceito em 10 de fevereiro de 2020. 


\section{Resumo}

O objetivo desta pesquisa foi analisar quais atributos influenciam a Satisfação geral dos acadêmicos do Curso de Contabilidade. Dessa forma, o referencial teórico utilizado para embasamento consistiu na satisfação dos estudantes e nas metodologias de ensino em Ciências Contábeis. Posteriormente, foi elaborado um background teórico para fundamentar a construção de nove hipóteses. Quanto à metodologia, a pesquisa é caracterizada pela abordagem quantitativa do tipo survey, com a coleta de dados obtidos de fonte primária, por meio da aplicação de questionários com escala Likert. A amostra da pesquisa é composta por 368 respondentes, acadêmicos dos Cursos de Ciências Contábeis matriculados em uma instituição de ensino superior privada situada na região Oeste do Estado do Paraná. Por fim, verificou-se que entre as categorias de análise propostas, todas demonstraram influência nos referidos constructos e, de forma positiva, exceto a Aprendizagem e o Desempenho pessoal. Além disso, identificou-se que os aspectos voltados à Qualidade do ensino/Desempenho do Curso, bem como os aspectos voltados ao Relacionamento com os professores são os que impactam a Satisfação geral com o Curso na percepção dos acadêmicos. Com isso, os achados evidenciaram a importância de uma percepção elevada sobre a Qualidade do ensino/Desempenho do Curso, na medida em que impacta significativamente na Satisfação geral do acadêmico.

Palavras-chave: Ciências Contábeis. Metodologias de ensino. Desempenho do Curso. Qualidade do ensino. Satisfação dos acadêmicos.

\section{Abstract}

The objective of this research is to analyze which attributes influence the General satisfaction of the students of the Course of Accounting. Thus, the theoretical framework used for the study consisted of students' satisfaction and teaching methodologies in Accounting Sciences. Subsequently, a theoretical background was elaborated to support the construction of nine hypotheses. Regarding the methodology, the research is characterized by the quantitative approach of the survey type, with the collection of data obtained from primary source, through the application of questionnaires with Likert scale. The research sample is made up of 368 respondents, academics from the Courses in Accounting Sciences enrolled in a private higher education institution located in the Western region of the State of Paraná. Finally, it was verified that among the categories of analysis proposed, all showed influence and in a positive way, except Learning and Personal performance. In addition, it was identified that aspects that focus on the Quality of teaching/Performance of the Course, and also aspects related to the relationship with teachers that impact the Overall Satisfaction with the Course in the perception of academics. With this, findings showed the importance of a high perception of the Quality of teaching/Course Performance, as it significantly impacts the Overall satisfaction of academics.

Keywords: Accounting. Teaching methodologies. Course Performance. Quality of teaching. Academics satisfaction.

\section{INTRODUÇÃO}

O aumento da oferta de serviços educacionais no Brasil é crescente e isso ocasiona aumento também na concorrência entre as Instituições de Ensino Superior (IES). Além disso, o cenário contábil apresenta-se envolto em uma série de transformações nas 
últimas décadas, de maneira que o ensino em Contabilidade se encontra em um estado de ressignificação (Beck \& Rausch, 2014). Pesquisadores como Andere e Araújo (2008) preconizam que a pesquisa sobre a temática da educação e do processo de ensinoaprendizagem contribui para a mudança e o desenvolvimento da sociedade. Cabe ressaltar que as organizações educacionais são consideradas uma das mais importantes em uma sociedade (Duong, 2015).

Nesse aspecto, as organizações, independentemente de seu ramo de atividade, necessitam se adaptar rapidamente para desenvolver uma gestão que atenda às expectativas e necessidades de seus clientes (Lizote, Verdinelli, Borba, \& Brasil, 2014). As IES, por sua vez, enfrentam um processo bem mais complexo que as demais organizações, em consequência de a natureza dos negócios ser classificada como serviços essencialmente intangíveis (Finger, 2000). Entretanto, de maneira semelhante aos outros tipos de organizações, o segmento de serviços educacionais posiciona-se pela perspectiva de que um elevado nível de qualidade disposto nos serviços prestados proporciona benefícios significativos em termos de participação de mercado, de fidelização e produtividade, entre outros aspectos relevantes (Maureira, 2004).

Diversas pesquisas no Brasil têm mostrado que a Satisfação dos estudantes se constitui uma variável fundamental para as IES na busca de um parâmetro adequado para a mensuração da qualidade dos serviços propostos na condição de prestador (Browne, Kaldenberg, Browne, \& Brown, 1998; Coda \& Silva, 2004; Duong, 2015; Gonçalves, Guerra, \& Moura, 2003; Guolla, 1999; Hermans, Haytko, \& Mott-Stenerson, 2009; Lizote et al., 2014; Lizote, Verdinelli, \& Lana, 2011; Mainardes \& Domingues, 2010; Mark, 2013; Marzo-Navarro, Pedraja-Iglesias, \& Rivera-Torres, 2005; Souki \& Pereira, 2004; Verdinelli, Souza, \& Tomio, 2009; Vieira, Milach, \& Huppes, 2008; Walter, 2006).

A satisfação se constitui, portanto, como um dos potenciais fatores para o comprometimento dos acadêmicos, bem como para a manutenção de uma imagem positiva da universidade (Gomes, Dagostini, \& Cunha, 2013). Dessa forma, com o intuito de reter e captar os estudantes, faz-se necessário que as IES realizem a medição interna de satisfação de alunos e professores. Nesse sentido, os estudos de Walter, Tontini e Domingues (2005) e Walter (2006) consideram a satisfação dos clientes não somente como resultado de um evento, mas também como produto de uma série de atividades e reações contínuas ao longo do tempo, perpassando dessa forma as maneiras clássicas de avaliação de produtos.

Adotando-se esse direcionamento como premissa, as IES buscam reforçar a qualidade de seus serviços prestados, agregando valor aos seus estudantes, consequentemente se tornando um notável diferencial competitivo (Carvalho, 2009; Lizote et al., 2014). Nessa perspectiva, o presente estudo torna-se relevante ao fomentar a discussão da melhoria $e$ aperfeiçoamento do processo de ensino-aprendizagem das IES e pretende responder à seguinte pergunta-problema: quais atributos influenciam a Satisfação geral dos acadêmicos 
do Curso de Ciências Contábeis? Assim, o objetivo deste estudo é analisar quais atributos influenciam a Satisfação geral dos acadêmicos do Curso de Ciências Contábeis de uma IES privada, localizada na região Oeste do Estado do Paraná. Com isso, a resposta desta pesquisa pode contribuir com os responsáveis pelo gerenciamento dos Cursos de Contabilidade, pois é uma das formas de reter os alunos e professores por meio do reconhecimento dos seus acadêmicos.

Destarte, a pesquisa é estruturada em cinco seções. Na primeira é apresentada a introdução, dispondo a contextualização, a problemática, a questão e os objetivos da pesquisa. A segunda seção apresenta a revisão da literatura, que versa a respeito da satisfação de estudantes e metodologias de ensino em Ciências Contábeis, além do background teórico para o embasamento das hipóteses trabalhadas. Na sequência são detalhados os procedimentos metodológicos empregados na pesquisa. Já na quarta seção são discutidas a descrição e a análise dos dados. Por fim, na última seção são apresentadas as considerações finais, e, depois delas, as referências utilizadas no texto.

\section{REVISÃO DA LITERATURA}

Esta seção apresenta a revisão da literatura utilizada para o desenvolvimento da pesquisa. São abordados os aspectos relacionados à satisfação dos estudantes e as metodologias de ensino em Ciências Contábeis, além do background teórico utilizado para o embasamento das hipóteses de pesquisa.

\subsection{SATISFAÇÃO DOS ESTUDANTES}

A concorrência estimula as organizações a inovarem e aumentarem a qualidade dos seus produtos e serviços que são ofertados ao mercado consumidor. Para tanto, a organização necessita desenvolver um trabalho que atenda às expectativas de sua clientela, embora seja considerado imperativo conhecer suas verdadeiras necessidades e desejos (Lizote et al., 2014). O ambiente no qual as instituições de ensino superior estão inseridas apresentou crescimento considerável nos últimos anos. Nesse sentido, a qualidade do ensino necessita de um planejamento pedagógico bem alinhado para não comprometer o desempenho dos discentes, proporcionando, dessa maneira, atuação profissional competitiva no mercado (Sousa, Nossa, Nascimento, \& Teixeira, 2017).

A satisfação dos acadêmicos é caracterizada como um aspecto fundamental para que se obtenha a lealdade e elementos consequentes, como, por exemplo, propaganda boca a boca e indicações, visto que o impacto da satisfação na lealdade é muito alto. Além disso, 
outro elemento que os gestores das IES precisam considerar também é que o processo de uso e consumo do serviço é longo, visto que os cursos possuem em média quatro anos (Gonçalves et al., 2003).

Para Paswan e Young (2002) a satisfação dos discentes é determinada por vários fatores, entre os quais: envolvimento do professor; interesse do aluno; interação professor e aluno; exigência do curso; e organização do curso. Dessa forma, a pesquisa sobre satisfação passou a ser mais abrangente. Seguindo a mesma linha, Carvalho (2009) argumenta que o sucesso das IES está relacionado com o material de apoio que elas utilizam para promover: interação; comprometimento e capacitação dos docentes dentro da sala de aula; a maneira como o curso está organizado; e questões relacionadas à infraestrutura da organização. Portanto, esses fatores são propostos para que as IES possam utilizá-los para avaliarem o desempenho de suas atividades, com a intenção de melhorar seus processos de aprendizagem relacionados aos aspectos de ensino, pesquisa, extensão e cultura geral (Lizote et al., 2014).

Semelhantemente, é possível verificar que as dimensões de qualidade, estrutura do curso, comprometimento e capacitação do corpo docente são fatores determinantes para que ocorra a satisfação e posterior fidelização dos estudantes às IES. Assim, as instituições necessitam utilizar métricas com a finalidade de verificar a satisfação dos estudantes, bem como realizar adaptações pedagógicas com o intuito de melhorar suas estruturas pedagógicas. Na próxima seção apresenta-se uma discussão sobre as metodologias de ensino em Ciências Contábeis.

\subsection{METODOLOGIAS DE ENSINO EM CIÊNCIAS CONTÁBEIS}

A Contabilidade é um campo do conhecimento científico que possui uma relação íntima com a sociedade e, nessa perspectiva, constitui-se uma área que requer atenção dos pesquisadores. Tradicionalmente, no Brasil, o ensino de Contabilidade é direcionado por uma abordagem normativista, isto é, orientado principalmente pelas normas e princípios da Contabilidade. Dessa maneira, o enfoque para as práticas contábeis se comporta timidamente, emergindo uma significativa resistência ao reconhecimento das pesquisas contábeis. Além disso, com o emprego de uma abordagem positivista, a pesquisa científica no campo da Contabilidade evidencia uma maior importância à habilidade do pesquisador com a utilização de ferramentas estatísticas e matemáticas, no propósito de testar hipóteses sobre o próprio conhecimento contábil em si (Dal Vesco, 2006).

Ao tratar das metodologias de ensino nos Cursos superiores de Ciências Contábeis, Pinto, Paula, e Gomes (2011) apontam os métodos passivos e os métodos ativos. Dessa forma, no método passivo de ensino o professor é caracterizado por ser o transmissor do conhecimento, 
uma vez que o conteúdo é proposto a priori e a sua exposição em sala de aula é considerada pontualmente. Com isso, a participação dos alunos nesse processo é praticamente limitada, provocando fortemente o desinteresse, a desmotivação e, consequentemente, o baixo nível de desempenho acadêmico (Pinto et al., 2011).

Nessa perspectiva, com a inserção de diferentes estratégias de ensino, Oliveira, Macêdo, Lira, e Pessoa (2012) comentam que o acadêmico, quando caracterizado por um papel ativo no processo de ensino-aprendizagem, desfruta de uma relação de troca de conhecimentos com o docente. Destaca-se ainda a importância de o docente conhecer e empregar os diferentes métodos de ensino e aprendizagem, proporcionando a construção de um ambiente interativo em sala de aula, uma vez que os alunos estarão cientes do que precisam aprender e o professor orientará o caminho para esse objetivo. Diante do exposto, a próxima seção demonstra o background que embasa as hipóteses da pesquisa em questão.

\subsection{BACKGROUND E HIPÓTESES DA PESQUISA}

Os estudos de Carvalho (2009) e Paswan e Young (2002) destacam aspectos relacionados à organização do curso como determinantes no desempenho deste. Lizote et al. (2014) verificaram que a organização do curso está positivamente relacionada com o envolvimento do professor, fato este que contribui para o desempenho. Em decorrência dessas relações, tem-se a primeira hipótese:

$\mathrm{H}_{1}:$ A organização da atividade do curso influencia de forma positiva a avaliação da Qualidade do ensino/Desempenho do Curso.

Os estudos de Mainardes \& Domingues (2010) e Walter (2006) identificaram a existência de relação positiva entre aspectos relacionados à grade curricular e à qualidade do ensino superior. Os achados de Paswan e Young (2002), por sua vez, indicaram que quanto maior a semelhança entre a teoria e a prática, maior será a percepção da qualidade de ensino por parte dos acadêmicos, sendo, portanto, necessário esse alinhamento dentro da grade curricular do curso. Com base nessas considerações, elaborou-se a segunda hipótese:

$\mathrm{H}_{2}$ : A Grade curricular influencia de forma positiva a avaliação da Qualidade do ensino/Desempenho do CURSO. 
O estudo dos autores Sousa et al. (2017) e Tucker, Jones, e Straker (2008) constataram relação positiva entre a ponderação adequada do professor sobre a avaliação do ensino superior, estando esta relacionada com o nível de exigência. No entanto, Costa, Chaves, e Pinheiro (2013) encontraram uma fraca correlação entre a grade curricular e a qualidade do ensino em Contabilidade. Partindo da consideração desses autores, tem-se a terceira hipótese:

$H_{3}: A$ avaliação adequada influencia de forma positiva o Nível de exigência do professor.

O estudo de Walter et al. (2005) e Walter (2006) destacam em seus achados a importância dos métodos de ensino utilizados pelos professores sobre as percepções de aprendizagem dos alunos. Os acadêmicos, nesses estudos, demonstraram a necessidade de receber conteúdos atualizados. Para Cavalcante e Santos (2013), o professor é o principal fator de desempenho satisfatório quando é conhecedor do assunto e explica o conteúdo apropriadamente. Por meio desses achados, constrói-se a quarta hipótese:

$\mathrm{H}_{4}$ : O Método de ensino influencia de forma positiva o Conteúdo atualizado.

Segundo Marks (2000), Walter (2006) e Wilhelm (2004), o nível de dificuldade afeta a percepção que os acadêmicos têm de sua aprendizagem. Os resultados destacam a importância do nível de exigência dos professores, de forma que se a exigência destes diminuir, a percepção de aprendizagem por parte dos alunos também será reduzida. Por meio desse raciocínio, tem-se a quinta hipótese:

$H_{5}$ : $O$ Nível de exigência do professor influencia de forma positiva a avaliação do Relacionamento com o professor.

As pesquisas de Morais, Santos, e Soares (2004), Walter et al. (2005) e Walter (2006) constataram a influência de aspectos relacionados com a atualização de conteúdos e professores sobre a percepção de aprendizagem dos alunos. Para Cavalcante e Santos (2013), o desempenho insatisfatório também é de responsabilidade do professor e é o principal fator, quando se detectam problemas com a explicação do conteúdo, com as provas aplicadas e no relacionamento entre alunos e professor. Existem outros fatores que estão relacionados ao estudante, como, por exemplo, a realização das atividades solicitadas. Por meio dessa constatação, apresenta-se a sexta hipótese: 
$\mathrm{H}_{6}$ : O Conteúdo atualizado influencia de forma positiva a avaliação da Aprendizagem percebida e desempenho pessoal.

Os achados de Walter (2006) identificaram que a qualidade do ensino impacta a avaliação de satisfação dos alunos. Além disso, os resultados encontrados pela respectiva autora evidenciaram que a qualidade se comportou como a variável de maior impacto na satisfação dos alunos, corroborando os estudos de Browne et al. (1998), Gonçalves et al. (2003), Guolla (1999), Mainardes \& Domingues (2010) e Marzo-Navarro et al. (2005).

Cabe ressaltar que, na perspectiva de Duong (2015), a satisfação dos acadêmicos é um fator significativo para a mensuração da qualidade, além de ser um aspecto fundamental para o sucesso das IES (Coda \& Silva, 2004; Ferraz et al., 2007; Hermans et al., 2009; Lizote et al., 2011; Souki \& Pereira, 2004; Verdinelli et al., 2009; Vieira et al., 2008). Nesse sentido, estudos apontam que as IES utilizam a satisfação como variável para a mensuração da qualidade (Hermans et al., 2009; Mark, 2013). Partindo dos achados desses autores, elaborou-se a sétima hipótese:

$\mathrm{H}_{7}$ : A Qualidade do ensino/Desempenho do Curso influencia de forma positiva a avaliação da Satisfação geral dos alunos com o curso.

O estudo de Lizote et al. (2014) constatou que a interação entre educando e educador se relaciona de maneira positiva com o interesse do aluno; além disso, foi verificado que o envolvimento do docente se relaciona positivamente com a Satisfação geral do aluno. Esses achados corroboram o estudo de Vieira et al. (2008), no qual se verificou que o envolvimento do professor e o interesse do estudante influenciam positivamente a Satisfação geral do Curso.

Paswan e Young (2002) destacam que a interação entre aluno e professor é um dos fatores determinantes para a aprendizagem e satisfação dos estudantes. Diante disso, os autores Calvo, Markauskaite, e Trigwell (2010), Dobransky e Frymier (2004) e Trigwell (2005) também verificaram uma relação positiva entre o relacionamento do professor com a Satisfação dos estudantes. Por meio dessa constatação, formulou-se a oitava hipótese:

$\mathrm{H}_{8}$ : $\mathrm{O}$ Relacionamento com o professor influencia de forma positiva a avaliação da Satisfação geral do Curso. 
Os achados de Marzo-Navarro et al. (2005) identificaram que o principal objetivo dos estudantes era aumentar seu nível de instrução. Walter (2006) demonstrou a relação entre aprendizagem percebida e satisfação dos acadêmicos. Ressalta-se, também, que tanto em nível nacional quanto em nível internacional existe um consenso referente à relação positiva entre estratégias de aprendizagem e um desempenho acadêmico melhor (QuevedoSilva, Lima-, Sauer, \& Reinert, 2012).

Todavia, quando o aluno detém um rendimento bom e que, entre outros aspectos, tenha conhecimento do seu grau de instrução, torna-se mais eficaz nas estratégias de aprendizagem (Holt, 1982).

Por outro lado, essa relação pode não ocorrer em Cursos de Contabilidade, por exemplo. Os achados de Nogueira (2009) demonstram que no Componente curricular de Contabilidade Gerencial os alunos não são afetados pelos estilos de aprendizagem. Porém, nem sempre os que mais aprendem são os que detêm o melhor desempenho (Washbush, \& Gosenpud, 1993). Entretanto, podem haver várias variáveis capazes de influenciar a opinião dos participantes, como ocorre com os níveis de satisfação e aprendizagem (Sauaia, 1995). Tendo como parâmetro esses estudos, fundamenta-se a nona hipótese:

$\mathrm{H}_{9}$ : A Aprendizagem percebida e desempenho pessoal influencia a avaliação da Satisfação geral dos alunos com o Curso.

A próxima seção demonstra os procedimentos adotados na presente pesquisa, desde a forma de coleta até a mensuração e a análise dos resultados.

\section{METODOLOGIA}

Este estudo foi desenvolvido por meio de uma pesquisa do tipo survey, com dados obtidos mediante fonte primária, em que a fonte de dados levantada se torna uma fonte permanente de informações (Babbie, 1999, Hair, Babin, Money, \& Samouel, 2005). Assim, a amostra deste estudo é caracterizada pelos estudantes dos Cursos de Ciências Contábeis, de uma instituição de ensino superior privada, situada na Cidade de Cascavel, região Oeste do Estado do Paraná.

Quanto à aplicação do instrumento, este foi impresso e distribuído in loco para as turmas presenciais. Dessa forma, perfizeram a quantidade de respondentes o montante de 368 acadêmicos. Diante disso, seis desses questionários tiveram ao menos uma questão sem resposta, sendo substituídos pela média da questão, com isso, não houve a necessidade de descarte de nenhum instrumento. Entendendo-se que a população dos alunos seguramente 
não passa de 500 acadêmicos, a Equação 1 demonstra o cálculo do erro amostral para uma precisão de $5 \%$ :

$$
n_{0}=\left(\frac{1}{0,05}\right)^{2}=400 \quad n=\frac{(500 \times 400)}{(500+400)}=222
$$

Assim, em razão de a amostra coletada ser maior que 222, a análise em questão detém um nível de precisão de, ao menos, $5 \%$ de significância. Por conseguinte, quanto ao instrumento aplicado, foi adaptado de Lizote et al. (2014), Sousa et al. (2017) e Walter (2006). A escala utilizada é a Likert, com diferencial semântico, com os polos antagônicos e a variância mínima de um e máxima de sete (Malhotra, 2006). Dessa forma, o questionário foi estruturado contendo 10 categorias de análise fundamentadas de acordo com as hipóteses.

A Figura 1 demonstra as categorias de análise da pesquisa, bem como as influências das dimensões:

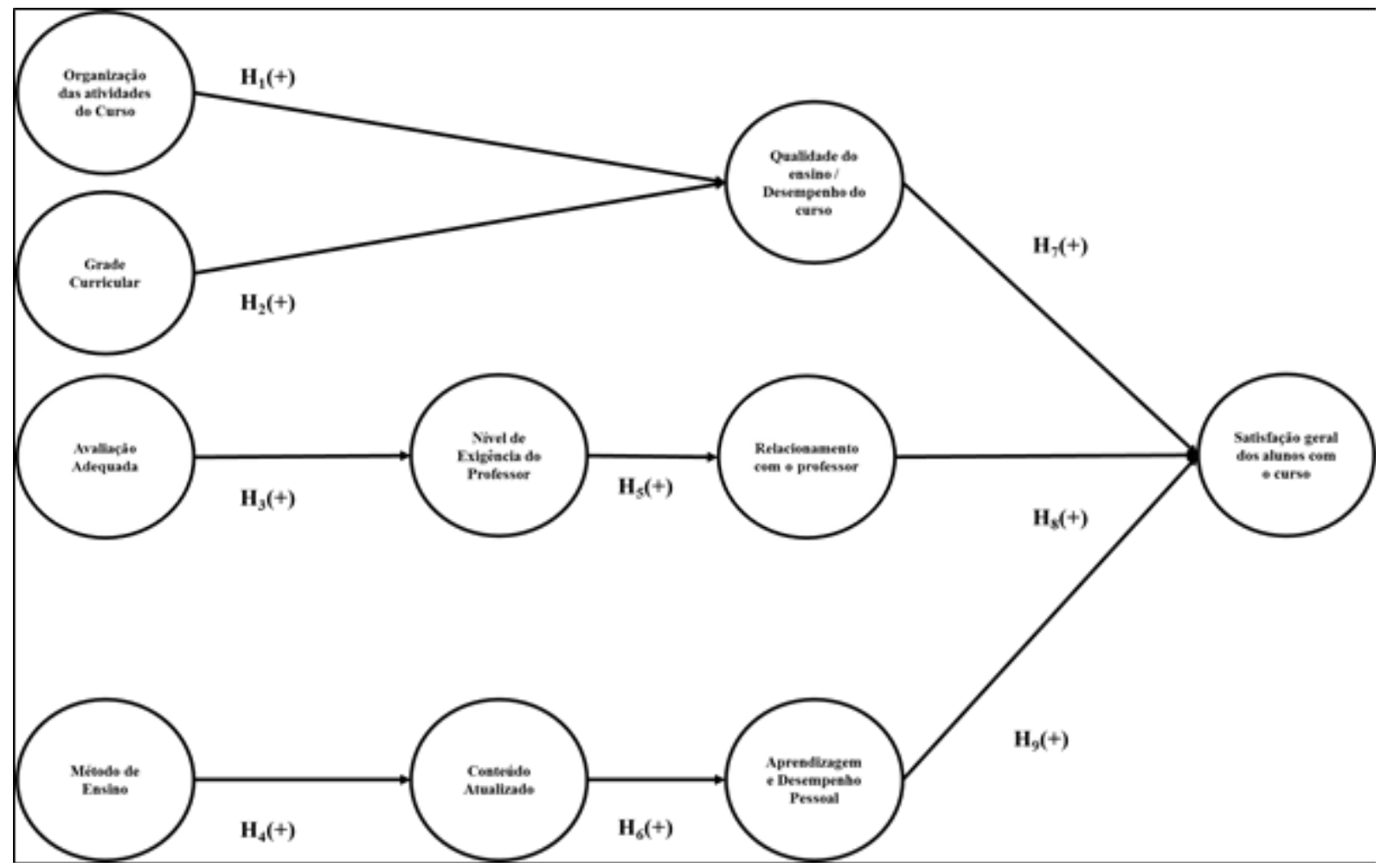

Figura 1. Influência hipotética do modelo de satisfação geral dos alunos. Adaptada de "Satisfação dos acadêmicos com o curso de Ciências Contábeis: Um estudo em Instituições de ensino superior privadas," by S. A. Lizote, M. A. Verdinelli, J. A. Borba, e M. L. A. V. Brasil, 2014, Advances in Scientific and Applied Accounting, 7, pp. 407-431, "Percepção de Qualidade do Curso de Ciências Contábeis: A Validação do Course Experience Questionaire com Estudantes Brasileiros," by W. D. Sousa, V. Nossa, J. C. H. B. Nascimento, e A. M. C. Teixeira, 2017, Enfoque Reflexão Contábil, 36, pp. 59-75 e "Antecedentes da satisfação e da lealdade de alunos de uma instituição de ensino superior," by S. A. Walter, 2006, (Dissertação de mestrado). Universidade Regional de Blumenau, Blumenau.

Ressalta-se que a análise foi realizada por meio das equações estruturais, pela flexibilidade em comparação aos demais métodos multivariados e por um teste confirmatório 
do instrumento em questão. Na segunda fase, foi realizado o teste com as dimensões agregadas ao instrumento aplicado.

Os procedimentos para a análise de dados se realizaram por meio de modelo e equações estruturais Strucutural Equation Modeling (SEM), conforme Geladi e Kowalski (1986), pela Equação 2:

$$
X=T P+E=\sum t h p h+E
$$

Diante disso, a pesquisa em questão é caracterizada como quantitativa, em decorrência da abordagem multivariada (Hair et al., 2005). Para o auxílio na organização e na análise dos dados, utilizou-se o software SmartPLS versão 3.2.6, além de planilhas eletrônicas. Portanto, na próxima seção são dispostas a apresentação e as discussões sobre os resultados encontrados na construção da pesquisa.

\section{APRESENTAÇÃO E DISCUSSÃO DOS RESULTADOS}

As equações estruturais empregadas nessa análise foram realizadas pelo método de PLS via bootstrapping, pois dessa maneira os coeficientes dos caminhos são mais estáveis e, por consequência, os $p$ valores são mais confiáveis, tanto para amostras pequenas quanto para amostras com outliers (Chiquoine, \& Hjalmarsson, 2009). Salienta-se também que o teste $t$ de Student é substituído com o uso do bootstrap (Azevedo et al., 2012), assim, foi analisado com 500 repetições. A amostra foi composta por 368 observações e 12 variáveis latentes, com 11 indicações. A Tabela 1 demonstra os testes estatísticos do modelo:

Tabela 1

Testes estatísticos do modelo

\begin{tabular}{lrrrr}
\hline \multicolumn{1}{c}{ Constructos } & $\begin{array}{c}\text { Alfa de } \\
\text { Cronbach }\end{array}$ & $\begin{array}{c}\text { Confiabilida- } \\
\text { de Composta }\end{array}$ & $\begin{array}{l}\text { Var. Média } \\
\text { Explicada }\end{array}$ & $\mathbf{r}^{\mathbf{2}}$ \\
\hline Aprendizagem e desempenho pessoal & 0,9546 & 0,9636 & 0,7328 & 0,8573 \\
\hline Conteúdo atualizado & 0,8544 & 0,8968 & 0,6376 & 0,4725 \\
\hline Avaliação adequada & 0,9880 & 0,9921 & 0,9767 & 0,7545 \\
\hline Grade curricular & 0,8564 & 0,8799 & 0,5339 & \\
\hline Método de ensino & 0,9553 & 0,9714 & 0,9189 & 0,9034 \\
\hline Nível de exigência do professor & 0,9849 & 0,9901 & 0,9708 & 0,9524 \\
\hline Organização do Curso & 0,9478 & 0,9663 & 0,9054 & 0,9698 \\
\hline $\begin{array}{l}\text { Qualidade do ensino/Desempenho do } \\
\text { Curso }\end{array}$ & 0,9270 & 0,9536 & 0,8728 & 0,8408 \\
\hline Relacionamento com o professor & 0,9681 & 0,9736 & 0,9755 & 0,9938 \\
\hline Satisfação geral & 0,9916 & 0 & & 0 \\
\hline
\end{tabular}


Os resultados da correlação dos constructos (variáveis latentes) obtiveram valores superiores a 0,7 , portanto, os dados estão adequados com relação ao Alfa de Cronbach (Hair et al., 2005). O coeficiente de Confiabilidade Composta também foi validado (Fornell \& Larcker, 1981), pois assim como o Alfa de Cronbach, também obteve valor acima de 0,7 para esse modelo. Dessa forma, foi possível realizar a validação do conteúdo estrutural (Burney, Henle, \& Widener, 2009), não se descartando nenhum constructo.

A Variância Média Explicada (VME) também demonstrou que a validade dos constructos foi convergente para a mensuração (Hair, Black, Babin, Anderson, \& Tatham, 2009), pois os resultados obtidos por ela foram maiores que 0,5. O menor valor obtido $(0,5339)$ refere-se ao constructo Grade curricular.

O modelo estrutural é validado pela verificação dos valores do $r^{2}$. A Aprendizagem e desempenho pessoal é explicada de forma direta pela dimensão de Conteúdo atualizado em $85,73 \%$. A dimensão de Conteúdo atualizado, por sua vez, é explicada em $47,25 \%$ de forma direta pelo Método de ensino; nota-se que a maior parte das variações dessa dimensão é determinada pelo erro. Assim, a percepção dos acadêmicos sobre o Método de ensino é explicada por outros fatores que não foram abordados no presente estudo.

O Nível de exigência do professor obteve o coeficiente de determinação igual a 75,45\% pela Avaliação adequada do conteúdo. As categorias de análise Organização das atividades do Curso e Grade curricular obtiveram um coeficiente de determinação igual a $90,34 \%$ sobre o constructo Qualidade do ensino/Desempenho do Curso. Já a categoria de análise Relacionamento com o professor deteve o coeficiente de determinação igual a 95,24\%, sendo este explicado pela categoria de Nível de exigência do professor.

A Satisfação geral apresentou o maior coeficiente de determinação, de maneira que as dimensões Qualidade do ensino/Desempenho do Curso, Relacionamento com o professor e Aprendizagem e desempenho pessoal possuem poder de explicação do constructo de $96,98 \%$. Entretanto, justifica-se esse valor em razão da quantidade de variáveis explicativas que compõe o modelo. A Tabela 2 demostra a validade discriminante dos constructos do presente estudo: 
Tabela 2

Validade discriminante

\begin{tabular}{|c|c|c|c|c|c|c|c|c|c|c|}
\hline $\begin{array}{l}\text { Categoria de aná- } \\
\text { lises }\end{array}$ & $\begin{array}{l}\text { Apd. } \\
\text { dp. p. }\end{array}$ & $\begin{array}{l}\text { Cont. } \\
\text { atual. }\end{array}$ & $\begin{array}{l}\text { Av. } \\
\text { adq. }\end{array}$ & $\begin{array}{l}\text { Gde. } \\
\text { cur. }\end{array}$ & $\begin{array}{l}\text { Mtdo. } \\
\text { ens. }\end{array}$ & $\begin{array}{l}\text { N. exi. } \\
\text { prof. }\end{array}$ & $\begin{array}{l}\text { Org. } \\
\text { Curso. }\end{array}$ & $\begin{array}{c}\text { Oldd. } \\
\text { Ens./Dp. }\end{array}$ & $\begin{array}{l}\text { Rel. } \\
\text { prof. }\end{array}$ & $\begin{array}{l}\text { Sat. } \\
\text { ger. }\end{array}$ \\
\hline $\begin{array}{l}\text { Aprendizagem e de- } \\
\text { sempenho pessoal }\end{array}$ &, 8560 & & & & & & & & & \\
\hline Conteúdo atualizado & ,9259 & 7985 & & & & & & & & \\
\hline Avaliação adequada & ,3715 & ,3040 & 9883 & & & & & & & \\
\hline Grade curricular & 6451 & ,5569 & 8040 & ,7307 & & & & & & \\
\hline Método de ensino & 6786 & 6874 & 2202 & ,4060 & 9586 & & & & & \\
\hline $\begin{array}{l}\text { Nível de exigência } \\
\text { do professor }\end{array}$ & 4224 & ,3657 & ,8686 &, 9142 & 2519 & 9853 & & & & \\
\hline $\begin{array}{l}\text { Organização do } \\
\text { Curso }\end{array}$ & ,4005 & ,3280 & ,8334 & 8668 & 2378 & 9362 & ,9515 & & & \\
\hline $\begin{array}{l}\text { Qualidade do ensi- } \\
\text { no/Desempenho do } \\
\text { Curso }\end{array}$ & 4104 & ,3362 & 8436 & ,8882 & 2438 & 9592 & 9386 & 9342 & & \\
\hline $\begin{array}{l}\text { Relacionamento com } \\
\text { o professor }\end{array}$ & 4175 &, 3421 & ,8582 & ,9235 & 2480 & 9759 & 9253 & 9481 & ,9169 & \\
\hline Satisfação geral & 4237 & ,3479 & 8802 & 9170 & ,2528 & 9897 & 9475 & 9621 & 9789 & ,9877 \\
\hline
\end{tabular}

Ressalta-se que as variáveis latentes que obtiveram indicadores próximos, ou com a correlação maior que a raiz quadrada da variância média extraída, não apresentam validade discriminante satisfatória. As variáveis latentes Aprendizagem e desempenho pessoal e Conteúdo atualizado apresentam indicadores bem próximos, isso pode indicar que o estudante tem maior percepção da aprendizagem quando lhe são apresentados Conteúdos atualizados (Walter et al., 2005; Walter, 2006).

O Conteúdo atualizado não detém validade discriminante sobre o Aprendizado e desempenho pessoal. De forma idêntica, as categorias Nível de exigência do professor, Organização do Curso, Qualidade do ensino/Desempenho do Curso, Relacionamento com o professor e Satisfação geral não apresentaram validade discriminante quando comparadas à Grade curricular. Os estudos de Walter (2006) e Mainardes \& Domingues (2010) identificaram a existência de relação positiva entre aspectos voltados à Grade curricular e à Qualidade do ensino superior.

A Satisfação geral e o Nível de exigência dos professores, com a Qualidade do ensino e Desempenho apresentaram indicadores bem próximos, o que também indica que não há validade discriminante entre as variáveis explanatórias e a independente. Evidenciase, dessa forma, que a Satisfação geral também está correlacionada com as suas variáveis explicativas, conforme Marks (2000), Marzo-Navarro et al. (2005), Nogueira (2009), Walter (2006) e Wilhelm (2004). Isso pode indicar que o estudante tem maior percepção sobre a satisfação com o curso quando possui uma relação mais próxima com o professor (Lizote et al., 2014; Paswan \& Young, 2002). 
Dessa forma, é necessário analisar se existe colinearidade entre as variáveis. A Tabela 3 demonstra a estatística de colinearidade (VIF) das variáveis independentes do modelo:

Tabela 3

Estatística de colinearidade (VIF) das variáveis independentes

\begin{tabular}{|c|c|c|c|c|c|c|}
\hline Constructo & $\begin{array}{l}\text { Aprendi- } \\
\text { zagem e } \\
\text { desempenho } \\
\text { pessoal }\end{array}$ & $\begin{array}{l}\text { Conteúdo } \\
\text { atualizado }\end{array}$ & $\begin{array}{l}\text { Nível de } \\
\text { exigência } p\end{array}$ & $\begin{array}{l}\text { Qualidade } \\
\text { do ensino/De- } \\
\text { sempenho do } \\
\text { curso }\end{array}$ & $\begin{array}{l}\text { Relaciona- } \\
\text { mento com } \\
\text { o professor }\end{array}$ & $\begin{array}{l}\text { Satisfação } \\
\text { geral }\end{array}$ \\
\hline Conteúdo atualizado & 1,000 & & & & & \\
\hline Avaliação adequada & & & 1,000 & & & \\
\hline Grade curricular & & & & 4,023 & & \\
\hline Método de ensino & & 1,000 & & & & \\
\hline $\begin{array}{l}\text { Nível de exigência } \\
\text { do professor }\end{array}$ & & & & & 1,000 & \\
\hline $\begin{array}{l}\text { Organização do } \\
\text { Curso }\end{array}$ & & & & 4,023 & & \\
\hline $\begin{array}{l}\text { Qualidade do ensi- } \\
\text { no/Desempenho do } \\
\text { Curso }\end{array}$ & & & & & & 9,921 \\
\hline $\begin{array}{l}\text { Relacionamento com } \\
\text { o professor }\end{array}$ & & & & & & 9,992 \\
\hline
\end{tabular}

Os índices da Tabela 3 demonstram apenas os valores referentes à colinearidade obtidos entre as variáveis por meio da Variance Inflation Factor (VIF). Com base nos valores apresentados, comprova-se a inexistência de colinearidade. Ressalta-se, portanto, que existe uma relação linear entre variáveis explicativas, pois os valores considerados aceitáveis ficam entre 1 e 10 (Corrar, Paulo, \& Dias, 2007).

Os valores menores encontrados foram inerentes às relações entre as seguintes variáveis: Conteúdo atualizado, Avaliação adequada, Método de ensino, Nível de exigência do professor, Relacionamento com o professor, todos iguais a 1. Já os valores máximos encontrados foram superiores a 9,9, referentes às variáveis: Relacionamento com o professor, Qualidade do ensino/Desempenho com o Curso e Satisfação geral.

As influências comprovadas e não comprovadas empregadas nas categorias de análise estão descritas na Figura 2: 


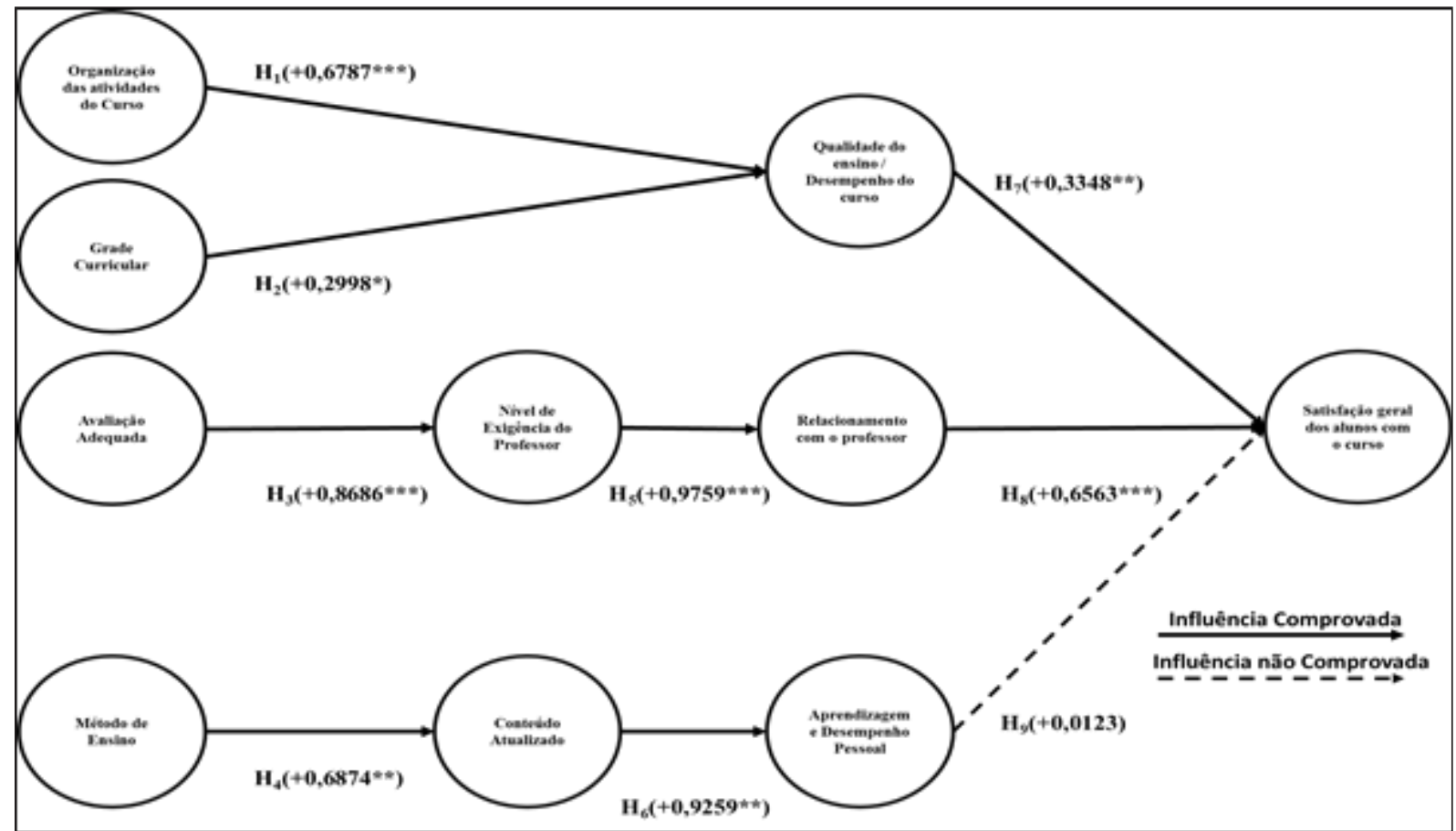

Figura 2. Coeficientes e influências encontradas para cada hipótese testada. Nota. ${ }^{* * *}$ p valor $<0,01,{ }^{* *}$ p valor $<0,05,{ }^{*}$ p valor $<0,1$.

De acordo com o $p$ valor encontrado $\left(H_{1}\right)$, a influência da Organização das atividades do Curso sobre a Qualidade do ensino/Desempenho do Curso não pode ser rejeitada ao nível de 1\%. Destarte, confirmam-se os resultados de Carvalho (2009), Lizote et al. (2014) e Paswan e Young (2002), visto que se constatou neste estudo uma relação positiva entre os aspectos relacionados à Organização do Curso com a dimensão Qualidade do ensino/ Desempenho do Curso.

Assim, essa influência sugere que a equipe responsável pela estruturação do Curso deve se ater ao planejamento das atividades dele, como, por exemplo: forma de agendamento dos trabalhos e avaliações e negociações de horários existentes entre os professores com o intuito de viabilizar outras atividades. Esses fatores influenciaram de forma positiva a percepção sobre a Qualidade do ensino/Desempenho do Curso na instituição de ensino pesquisada, quando analisados na percepção dos acadêmicos de Ciências Contábeis.

A influência da Grade curricular sobre a Qualidade do ensino/Desempenho do Curso $\left(\mathrm{H}_{2}\right)$ não pode ser rejeitada ao nível de $10 \%$. Dessa forma, os resultados encontrados estão em harmonia com os estudos de Mainardes \& Domingues (2010), Paswan e Young (2002) e Walter (2006), demonstrando que para os acadêmicos do Curso de Contabilidade também têm a mesma influência.

A percepção dos acadêmicos referente aos aspectos voltados à Grade curricular, como cargas horárias, ordenamento das disciplinas, direcionamento do Curso e qualidade e relevância das disciplinas determinam a percepção sobre desempenho do Curso em 
comparação com outras instituições, porém não influenciam a percepção sobre o atendimento às suas necessidades pessoais, nem a percepção sobre a qualidade geral do Curso.

Nota-se também que as categorias de análises Organização das atividades do Curso e Grade curricular, fundamentadas para explicar a percepção sobre a Qualidade do ensino/ Desempenho do Curso foram validadas, permanecendo em consonância com os estudos que as fundamentaram.

A influência da Qualidade do ensino/Desempenho do Curso sobre a Satisfação geral com o Curso $\left(\mathrm{H}_{7}\right)$, além de significância de $5 \%$, obteve influência positiva. Assim, os resultados encontrados vão de encontro aos estudos de Gonçalves et al. (2003), que descrevem que a Qualidade é a que mais impacta na Satisfação.

Diante disso, quanto maior a percepção dos acadêmicos sobre o Curso de Contabilidade da instituição em comparação com as outras instituições, maior será a satisfação geral destes. Da mesma forma, quanto maior a percepção de que o Curso atende à sua necessidade, maior será a Satisfação geral com o Curso. Na mesma direção, ressalta-se a percepção de qualidade geral do Curso com a Satisfação geral.

A influência da Avaliação adequada sobre a Qualidade do ensino/Desempenho do Curso $\left(\mathrm{H}_{3}\right)$ não pode ser rejeitada ao nível de significância de $10 \%$, uma vez que se demonstra positiva. Assim, esse resultado corrobora os achados de Sousa et al. (2017) e Tucker et al. (2008), nos quais se destacaram a importância da avaliação adequada sobre o aprendizado dos alunos.

Esse resultado sugere que quanto mais os professores testam os conhecimentos dos alunos com o intuito de realmente certificar se houve entendimento e memorização, maior será a percepção sobre o nível de exigência dos docentes. Assim, quanto mais os professores antecipam problemas ensinados, mais exigentes se tornam, isto é, quando desafiam os alunos com situações que ainda não ocorreram, por exemplo. Essas percepções justificam-se pelo fato de que o professor retira os alunos da zona de conforto, estimulando-os a buscarem novos desafios.

Nesse sentido, a influência do Nível de exigência dos professores $\left(H_{5}\right)$ exerce uma influência positiva, ao nível de 1\% de significância, na percepção sobre o Relacionamento com os professores. Com isso, os resultados de Marks (2000), Walter (2006) e Wilhelm (2004) também estão em consenso com os resultados alcançados neste estudo. Tais resultados indicam que quanto maior for a percepção sobre a flexibilidade com relação aos trabalhos e provas, bem como quanto aos critérios justos de avaliações e ao nível de exigência, considerando-se o conteúdo ministrado, maior será a percepção sobre o relacionamento do acadêmico com o professor.

O Relacionamento com o professor $\left(\mathrm{H}_{8}\right)$ também demonstrou influência positiva e estatisticamente significante sobre a Satisfação geral, contudo, ao nível de 1\% de significância. 
Assim, os achados de Lizote et al. (2014) também estão em harmonia com o presente estudo, pois foi constatada uma interação positiva nesse relacionamento. Os autores Paswan e Young (2002) também ressaltam que essa interação é uma satisfação dos acadêmicos.

O Método de ensino influencia positivamente o Conteúdo atualizado $\left(\mathrm{H}_{4}\right)$, ao nível de significância $5 \%$. Assim, os resultados estão em harmonia com os resultados encontrados por Walter et al. (2005) e Walter (2006), que notaram a importância dos métodos de ensino perante as percepções sobre a aprendizagem dos alunos.

Conforme as unidades de análise dessa categoria, pode-se inferir que quanto maior a percepção sobre a clareza dos assuntos expostos com relação ao Domínio dos professores a respeito do conteúdo e da utilização de Métodos de ensino adequados, maior será a percepção dos acadêmicos com relação ao contexto no qual estão inseridos. Evidenciase que no componente curricular, ter o Conteúdo atualizado influencia positivamente $\left(\mathrm{H}_{6}\right)$ a percepção dos acadêmicos sobre a sua Aprendizagem e desempenho pessoal, com significância de $5 \%$. Em consonância com esses achados, estão os resultados discutidos por Walter et al. (2005) e Walter (2006).

Essa influência justifica-se pelo fato de que quanto mais os professores estão a tualizados e detêm mais especializações em nível stricto sensu, mais demonstram conhecimento sobre os assuntos ministrados. Esse fator impacta tanto a percepção dos acadêmicos sobre a Aprendizagem quanto a percepção sobre o Desempenho pessoal.

Todavia, a Aprendizagem percebida e Desempenho pessoal não influenciam $\left(H_{9}\right)$ a Satisfação geral com o Curso. Esses resultados divergem dos estudos de Marzo-Navarro et al. (2005) e Walter (2006). Essa relação justifica-se pelo fato de os alunos terem a percepção de que o nível de aprendizagem é algo que não compete à instituição e aos professores.

De acordo com as categorias de análise propostas, a Satisfação geral do Curso foi explicada pelos aspectos voltados ao professor e pela Qualidade de ensino/Desempenho do Curso. Destarte, os aspectos voltados ao Relacionamento com os professores são os que mais influenciam a percepção dos alunos em comparação ao planejamento das atividades pelo nível de significância e pelos valores dos coeficientes.

\section{CONSIDERAÇÕES FINAIS}

O presente estudo objetivou analisar quais atributos influenciam a Satisfação geral dos acadêmicos do Curso de Contabilidade. Entre as categorias de análise propostas, todas se demonstraram como influência positiva, exceto a Aprendizagem e Desempenho pessoal. Destaca-se, também, que foram identificados aspectos voltados à Qualidade do ensino/ 
Desempenho do Curso, bem como aspectos voltados ao Relacionamento com os professores, os quais impactam a Satisfação geral com o Curso na percepção dos acadêmicos.

Ao analisar sobre o foco da Qualidade do ensino/Desempenho do Curso, foram encontrados resultados que sugerem que esses atributos são influenciados pela percepção sobre a organização e planejamento das atividades deste. Dessa forma, a maneira como são organizadas as atividades do Curso e o planejamento das atividades são fatores determinantes nos quais os gestores das instituições de ensino devem se ater.

Ressalta-se que a percepção sobre a Grade curricular também influencia de maneira similar a Qualidade do ensino/Desempenho do Curso. Esses resultados sugerem que os acadêmicos do Curso de Contabilidade reconhecem a importância da Grade curricular elaborada pela instituição, percepção esta que impacta positivamente a Qualidade do ensino/ Desempenho do Curso. Assim, para conseguir aumentar a percepção sobre a Qualidade do ensino, sugere-se aos gestores a criação de mecanismos para elevar a importância percebida do componente curricular, como relacioná-lo com a teoria e a prática, por exemplo.

A importância de se ter uma percepção elevada sobre a Qualidade do ensino/ Desempenho do curso está no impacto que ela causa na Satisfação geral do acadêmico com - Curso. Assim, se essas relações forem observadas pelos gestores das instituições, poderão contribuir de forma eficiente na satisfação dos acadêmicos.

Por outro lado, o professor também ocupa um papel de destaque na percepção do aluno perante a Satisfação geral, pois os resultados demonstraram que quanto mais os professores instigam os alunos na busca do aprendizado, maior será o Nível de exigência. Porém, mesmo que essas atitudes retirem os alunos da zona de conforto que se instaura na sala de aula, se forem elaboradas de forma adequada, impactam diretamente a Satisfação geral dos acadêmicos.

Como contribuição, nesta pesquisa enfatiza-se que para a tomada de decisão cabe aos gestores dos cursos observarem que a percepção dos acadêmicos com relação à Satisfação geral tem como determinante a postura dos professores. Dessa forma, atrair e reter profissionais com as habilidades para gerenciar as avaliações e o nível de exigência considerando o grau de dificuldade dos conteúdos é uma forma de aumentar a Satisfação geral dos acadêmicos.

Todavia, esses aspectos não podem limitar os professores no desempenho das atividades de avaliação. Assim, esses resultados apontam que pode haver uma balança entre a Satisfação do Curso e as habilidades dos professores, desde o Nível de exigência até a forma de avaliação, bem como no Domínio do conteúdo e especialização na área ministrada. Além disso, esses resultados descrevem que quanto mais os professores são atualizados, mais capazes são de influenciar a percepção dos alunos com relação à Satisfação geral com o Curso. Destaca-se, ainda, que no que se refer à tomada de decisão dos gestores, atrair e 
reter esses profissionais especializados pode ser considerada uma estratégia para aumentar a Satisfação geral.

A limitação desta pesquisa está no fato de ter sido aplicada a apenas uma instituição de ensino, bem como serem observadas apenas as percepções dos acadêmicos do Curso de Contabilidade. Entretanto, para futuras pesquisas, sugere-se uma abordagem qualitativa para se analisar com mais profundidade a percepção de aprendizagem percebida dos acadêmicos com a Satisfação geral do Curso.

\section{REFERÊNCIAS}

Andere, M. A., \& Araújo, A. M. P. (2008). Aspectos da formação do professor de ensino superior de Ciências Contábeis: Uma análise dos programas de pós-graduação. Revista Contabilidade e Finanças, 19(48), 91-102.

Azevedo, C. E. F, Oliveira, L. G. L, Abdalla, M. M., Gonzalez, R. K., Ribeiro, A. J. G., \& Holperin, M. M. (2012). Por que Finanças? Avaliando o interesse dos estudantes de graduação em administração pela área de finanças. RAM. Revista de Administração Mackenzie, 13(6).

Babbie, E. (1999). Métodos de pesquisas de survey. Belo Horizonte: UFMG.

Beck, F., \& Rausch, R. B. (2014). Fatores que influenciam o processo ensino-aprendizagem na percepção de discentes do curso de ciências contábeis. Contabilidade Vista \& Revista, 25(2), 38-58.

Browne, B. A., Kaldenberg, D. O., Browne, W. G., \& Brown, D. J. (1998). Student as customer: Factors affecting satisfaction and assessments of institutional quality. Journal of Marketing for Higher Education, 8(3), 1-14.

Burney, L. L., Henle, C. A., \& Widener, S. K. (2009). A path model examining the relations among strategic performance measurement system characteristics, organizational justice, and extra-and in-role performance. Accounting, Organizations and Society, 34(3), 305-321.

Calvo, R. A., Markauskaite, L., \& Trigwell, K. (2010). Factors affecting students' experiences and satisfaction about teaching quality in engineering. Australasian Journal of Engineering, 16, 325-338.

Carvalho, R. J. F. (2009). Os factores determinantes da satisfação dos alunos de mestrados de continuidade (Dissertação de mestrado). Instituto Superior de Ciências do Trabalho e da Empresa, Lisboa, Portugal. 
Cavalcante, C. H. L., \& Santos, P. A., Jr. (2013). Fatores que influenciam o desempenho escolar: A percepção dos estudantes do curso Técnico em Contabilidade do IFRS-Instituto Federal de Educação, Ciência e Tecnologia do Rio Grande do Sul, Campus Porto Alegre. Revista Liberato, 14(21), 29-50.

Chiquoine, B., \& Hjalmarsson, E. (2009). Jackknifing stock return predictions. Journal of Empirical Finance, 16(5), 793-803.

Coda, R. \& Silva, D. (2004). Sua escola de Administração é uma excelente escola para se estudar? Descobrindo dimensões da satisfação de alunos em cursos de Administração: Uma contribuição metodológica. Anais do Encontro da Anpad, Curitiba, 28.

Corrar, L. J., Paulo, E., \& Dias, J. M., Filho. (2007). Análise multivariada: Para os cursos de administração, ciências contábeis e economia. São Paulo: Atlas.

Costa, A. Q., Chaves, A. N. P., \& Pinheiro, V. L. F. (2013). A grade curricular dos cursos de Ciências Contábeis e os resultados institucionais no ENADE: Um estudo nas disciplinas da área da contabilidade financeira e de orçamento. Revista Gestão Universitária na América Latina-GUAL, 6(3), 127-145.

Dal Vesco, D. G. (2006). O ensino de contabilidade no Brasil. CAP Accounting and Management, $1(1), 80-84$.

Dobransky, N. D., \& Frymier, A. B. (2004). Developing teacher-student relationships through out of class communication. Communication Quarterly, 52, 211-223.

Duong, M. Q. (2015). The factors influencing student satisfaction in Vietnamese higher education. International Research in Education, 4(1), 27-38.

Ferraz J. J., Souza, M. J. B., \& Verdinelli, M. A. (2007). Percepção da imagem e satisfação em egressos universitários: Uma análise correlacional. Anais do Colóquio Internacional sobre Gestão Universitária da América do Sul, Mar del Plata, Argentina, 7.

Finger, A. B. (2000). A qualidade dos cursos de mestrado em administração: Uma avaliação pela percepção discente (Dissertação de mestrado). Universidade Federal de Santa Catarina, Florianópolis.

Fornell, C., \& Larcker, D. F. (1981). Evaluating structural equation models with unobservable variables and measurement error. Journal of marketing research, 18(1), 39-50. 
Geladi, P., \& Kowalski, B. R. (1986). Partial least-squares regression: A tutorial. Analytica chimica acta, 185, 1-17.

Gomes, G., Dagostini, L., \& da Cunha, P. R. (2013). Satisfação dos Estudantes do Curso de Ciências Contábeis: Estudo em uma faculdade do Paraná. Revista da Faculdade de Administração e Economia, 4(2), 102-123.

Gonçalves, C., Filho, Guerra, R. S., \& Moura, A. (2003). Mensuração de satisfação, qualidade, lealdade, valor e expectativa em instituições de ensino superior: Um estudo do modelo ACSI através de equações estruturais. Anais do Encontro Nacional dos Programas de Pós-graduação em Administração, Atibaia, 27.

Guolla, M. (1999). Assessing the teaching quality to student satisfaction relationship: Applied customer satisfaction research in the classroom. Journal of Marketing Theory and Practice, 7(3), 87-97.

Hair, J. F., Jr., Babin, B., Money, A. H., \& Samouel, P. (2005). Fundamentos de métodos de pesquisa em administração. Porto Alegre: Bookman.

Hair, J. F., Jr., Black, W. C., Babin, B. J., Anderson, R. E., \& Tatham, R. L. (2009). Análise multivariada de dados. ( $6^{a}$ ed.). Porto Alegre: Bookman.

Hermans, C. M., Haytko, D. L., \& Mott-Stenerson, B. (2009). Student Satisfaction in Web-Enhanced Learning Environments. Journal of instructional pedagogies, 1.

Holt, J. (1982). How children fail. New York: Delta.

Lizote, S. A., Verdinelli, M. A., Borba, J. A., \& Brasil, M. L. A. V. (2014). Satisfação dos acadêmicos com o curso de Ciências Contábeis: Um estudo em Instituições de ensino superior privadas. Advances in Scientific and Applied Accounting, 7(3), 407-431.

Lizote, S. A., Verdinelli, M. A., \& Lana, J. (2011). Satisfação dos alunos dos cursos de pós-graduação lato sensu: Um estudo através da modelagem em equações estruturais. Anais do Colóquio Internacional sobre Gestão Universitária de América do Sul, Florianópolis, 11.

Mainardes, E. W., \& Domingues, M. J. C. S. (2010). Satisfação de estudantes em administração de Joinville/SC. Revista Pensamento Contemporâneo em Administração, 4(2). 
Malhotra, N. K. (2006). Pesquisa de marketing: Uma orientação aplicada. Porto Alegre: Bookman.

Mark, E. (2013). Student satisfaction and the customer focus in higher education. Journal of Higher Education Policy and Management, 35(1), 210.

Marks, R. B. (2000). Determinants of student evaluations of global measures of instructor and course value. Journal of Marketing Education, 22(2), 108-119.

Marzo-Navarro, M., Pedraja-Iglesias, M., \& Rivera-Torres, M. (2005). Measuring customer satisfaction in summer courses. Quality Assurance in Education, 13(1), 53-65.

Maureira, O. (2004). El liderazgo factor de eficacia escolar, hacia un modelo causal. REICE-Revista Iberoamericana sobre Calidad, Eficacia y Cambio en Educación, 2(1).

Morais, J. J. S., Santos, C. M. L. D., \& Soares, T. A. S. (2004). Ensino da Contabilidade: Uma análise crítica. Pensar Contábil, 6(24), 12-16.

Nogueira, D. R. (2009). O impacto do estilo de aprendizagem no desempenho acadêmico: Um estudo empírico com alunos das disciplinas de contabilidade geral e gerencial na educação à distância (Dissertação de mestrado). Universidade Federal do Paraná, Curitiba.

Oliveira, L. C. G., Macêdo, J. M. A., Lira, I. A., \& Pessoa, L. G. D. S. B. (2012). Metodologias de Ensino Superior: Uma análise de sua utilização pelos professores do Curso de Ciências Contábeis da Universidade Federal de Campina Grande-Campus de Sousa-PB. SINERGIA-Revista do Instituto de Ciências Econômicas, Administrativas e Contábeis, 15(1), 37-50.

Paswan, A. K., \& Young, J. A. (2002). Student evaluation of instructor: A nomological investigation using structural equation modeling. Journal of Marketing Education, 24(3), 193 202.

Pinto, P. S. B., Paula, M. M. D., \& Gomes, J. S. (2011). Metodologia do ensino: Uma análise da percepção dos alunos frente a diferentes formas de ensino. Sociedade, Contabilidade e Gestão, 6(3).

Quevedo-Silva, F., Lima-, D. O., Filho, Sauer, L., \& Reinert, J. N. (2012). Fatores discriminantes no grau de satisfação de estudantes de Administração. Revista de Economia e Administração, 11(1), 28-45. 
Sauaia, A. C. A. (1995). Satisfação e aprendizagem em jogos de empresas: Contribuições para a educação gerencial (Tese de doutorado). Universidade de São Paulo, São Paulo.

Souki, G. Q., \& Pereira, C. A. (2004). Satisfação, motivação e comprometimento de estudantes de administração: Um estudo com base nos atributos de uma instituição de ensino superior. Anais do Encontro Nacional dos Programas de Pós-Graduação em Administração, Curitiba, 28.

Sousa, W. D., Nossa, V., Nascimento, J. C. H. B., \& Teixeira, A. M. C. (2017). Percepção de Qualidade do Curso de Ciências Contábeis: A Validação do Course Experience Questionaire com Estudantes Brasileiros. Enfoque Reflexão Contábil, 36(2), 59-75.

Trigwell, K. (2005). Teaching-research relations, cross-disciplinary collegiality and student learning. Higher Education, 49, 235-254.

Tucker, B., Jones, S., \& Straker, L. (2008). Online student evaluation improves Course Experience Questionnaire results in a physiotherapy program. Higher Education Research \& Development, 27(3), 281-296.

Verdinelli, M. A., Souza, M. J. B. D., \& Tomio, J. L. (2009). Análise da relação entre a imagem institucional e a satisfação dos alunos para subsidiar o marketing educacional. Anais do Colóquio Internacional sobre Gestão Universitária de América do Sul, Florianópolis, 9.

Vieira, K. M., Milach, F. T., \& Huppes, D. (2008). Equações estruturais aplicadas à satisfação dos alunos: Um estudo no Curso de Ciências Contábeis da Universidade Federal de Santa Maria. Revista Contabilidade \& Finanças-USP, 19(48), 65-76.

Walter, S. A. (2006). Antecedentes da satisfação e da lealdade de alunos de uma instituição de ensino superior (Dissertação de mestrado). Universidade Regional de Blumenau, Blumenau.

Walter, S. A., Tontini, G., \& Domingues, M. J. C. S. (2005). Identificando oportunidades de melhoria em um curso superior através da análise da satisfação dos alunos. Anais do Encontro Nacional de Programas de Pós-Graduação em Administração, Brasília, DF, 29.

Washbush, J., \& Gosenpud, J. (1993). The relationship between total enterprise simulation performance and learning. Developments in Business Simulation and Experiential Learning, 20. 
Wilhelm, W. B. (2004). The relative influence of published teaching evaluations and other instructor attributes on course choice. Journal of Marketing Education, 26(1), 17-30.

\section{Como citar este artigo:}

\section{ABNT}

FERREIRA, Rafael Maximiano; FERREIRA, Rafael Maximiano; LIMA, Samuel Lyncon Leandro de. Satisfação dos acadêmicos de Ciências Contábeis: Um estudo com equações estruturais. RACE, Revista de Administração, Contabilidade e Economia, Joaçaba: Editora Unoesc, v. 19, n. 1, p. 75-98, jan./abr. 2020. Disponível em: http://editora.unoesc.edu.br/index. php/race. Acesso em: dia/mês/ano.

\section{APA}

Ferreira, R. M., Ferreira, R. M., \& Lima, S. L. L. de. (2020). Satisfação dos acadêmicos de Ciências Contábeis: Um estudo com equações estruturais. RACE, Revista de Administração, Contabilidade e Economia, 19(1), 75-98. Recuperado de http://editora.unoesc.edu. br/index.php/race 